\title{
Glycaemic impact of patient-led use of sensor-guided pump therapy in type 1 diabetes: a randomised controlled trial
}

\author{
M. A. O'Connell • S. Donath • D. N. O'Neal • \\ P. G. Colman • G. R. Ambler • T. W. Jones • \\ E. A. Davis • F. J. Cameron
}

Received: 14 December 2008 / Accepted: 25 March 2009/Published online: 25 April 2009

(C) Springer-Verlag 2009

\begin{abstract}
Aims/hypothesis The objective of this study was to assess the impact of patient-led sensor-guided pump management on glycaemic control, and compare the effect with that of standard insulin pump therapy.

Methods An open multicentre parallel randomised controlled trial was conducted at five tertiary diabetes centres. Participants aged 13.0-40.0 years with well-controlled type
\end{abstract}

M. A. O'Connell • F. J. Cameron $(\bowtie)$

Department of Endocrinology and Diabetes, The Royal Children's Hospital Melbourne and Murdoch Childrens Research Institute,

Flemington Road, Parkville,

Melbourne, VIC 3052, Australia

e-mail: fergus.cameron@rch.org.au

S. Donath

Clinical Epidemiology and Biostatistics Unit,

The Royal Children's Hospital Melbourne,

and Murdoch Childrens Research Institute,

Melbourne, VIC, Australia

D. N. O'Neal

Department of Medicine, St Vincent's Hospital Melbourne,

Melbourne, VIC, Australia

P. G. Colman

Department of Diabetes and Endocrinology,

Royal Melbourne Hospital,

Melbourne, VIC, Australia

G. R. Ambler

Institute of Endocrinology and Diabetes,

The Children's Hospital at Westmead,

Sydney, NSW, Australia

T. W. Jones $\cdot$ E. A. Davis

Department of Endocrinology and Diabetes,

Princess Margaret Hospital for Children,

Perth, WA, Australia
1 diabetes were randomised 1:1 to either study group for 3 months. Randomisation was carried out using a central computer-generated schedule. Participants in the intervention group used sensor-guided pump management; no instructive guidelines in interpreting real-time data were provided ('patient-led' use). Participants in the control group continued their original insulin pump regimen. Continuous glucose monitoring (CGM) and $\mathrm{HbA}_{1 \mathrm{c}}$ level were used to assess outcomes. The primary outcome was the difference in the proportion of time in the target glycaemic range during the 3 month study period (derived from CGM, target range 4-10 $\mathrm{mmol} / \mathrm{l}$ ). Secondary outcomes were difference in $\mathrm{HbA}_{1 \mathrm{c}}$, time in hypoglycaemic $(\leq 3.9 \mathrm{mmol} / \mathrm{l})$ and hyperglycaemic $(\geq 10.1 \mathrm{mmol} / \mathrm{l})$ ranges and glycaemic variability.

Results Sixty-two participants were recruited and randomised; 5/31 and 2/31 withdrew from intervention and control groups, respectively, leaving 26/31 and 29/31 for the intention-to-treat analyses. When adjusted for baseline values, the mean end-of-study $\mathrm{HbA}_{1 \mathrm{c}}$ was $0.43 \%$ lower in the intervention group compared with the control group (95\% CI 0.19 to $0.75 \% ; p=0.009)$. No difference was observed in CGM-derived time in target (measured difference $1.72 ; 95 \% \mathrm{CI}-5.37$ to 8.81$)$, hypoglycaemic $(0.54$; $95 \%$ CI -3.48 to 4.55$)$ or hyperglycaemic $(-2.18 ; 95 \%$ CI -10.0 to 5.69$)$ range or in glycaemic variability $(-0.29$; $95 \%$ CI -0.34 to 0.28 ). Within the intervention group, $\mathrm{HbA}_{1 \mathrm{c}}$ was $0.51 \%$ lower in participants with sensor use $\geq 70 \%$ compared with participants with sensor use $<70 \%$ (95\% CI -0.98 to $-0.04, p=0.04)$. Five episodes of device malfunction occurred.

Conclusions/interpretation Individuals established on insulin pump therapy can employ sensor-guided pump management to improve glycaemic control. An apparent dose-dependent effect of sensor usage was noted; however, 
frequent use of this technology $(\geq 70 \%)$ was not universally acceptable.

Trial registration: ACTRN12606000049572

Funding: Funding support and equipment were provided by Medtronic Australasia.

Keywords Clinical diabetes · Devices · Insulin-infusion systems · Insulin therapy · Randomised controlled trial .

Type 1 diabetes

$\begin{array}{ll}\text { Abbreviations } \\ \text { CGM } & \text { Continuous glucose monitoring } \\ \text { CONGA } & \text { Continuous overlapping net glycaemic action } \\ \text { MARD } & \text { Mean absolute relative difference } \\ \text { RCT } & \text { Randomised controlled trial } \\ \text { RT } & \text { Real time } \\ \text { RT-CGM } & \text { Real-time continuous glucose monitoring } \\ \text { SMBG } & \text { Self-monitoring of blood glucose }\end{array}$

The advent of continuous glucose monitoring (CGM) has allowed significant further insights into diabetes control. Initial CGM devices were predominantly clinicianorientated, as recorded data were only accessible retrospectively. Use of these devices to initiate focused therapeutic changes has been studied extensively with some [1-4], but not all $[5,6]$ studies reporting improvements in overall glycaemic control.

In contrast, the newest generation of CGM devices are patient-orientated and offer real-time (RT) access to interstitial glucose levels. Studies assessing their use have shown improvement in $\mathrm{HbA}_{1 \mathrm{c}}$ [7-11], increased time spent in the target glucose range $[11,12]$ and fewer hypo- and hyperglycaemic excursions $[9,13]$, suggesting the potential for important clinical benefits.

Patients using insulin pump therapy may gain maximal benefit from real-time continuous glucose monitoring (RTCGM), as continuous subcutaneous insulin delivery can be altered in response to real-time glucose trends. Use of RTCGM with insulin pump therapy can be described as sensor-guided pump management. The MiniMed Paradigm REAL-Time system (Medtronic MiniMed, Northridge, CA, USA) integrates RT-CGM with insulin pump technology. Interstitial glucose readings are transmitted and displayed on the device screen every $5 \mathrm{~min}$ for up to $72 \mathrm{~h}$; glucosetrend information and programmable hypo- and hyperglycaemic alarms are further features. Of note, RT data recorded and displayed by the system have no inherent influence on insulin delivery; rather, the need for therapeutic changes must be recognised and initiated by the user.

Previous controlled studies of RT-CGM technology [8, $10,14]$ have incorporated regular clinician review of the RT data and clinician-led adjustments to insulin delivery, which may have impacted on the glycaemic improvements accrued. Whether individuals with type 1 diabetes can adapt to and employ RT-CGM to improve their own glycaemic control has not previously been tested. The aim of this study, therefore, was to assess the impact of patientled use of sensor-guided pump management on indices of glycaemic control in adolescents and young adults with type 1 diabetes and compare the impact with that of standard insulin pump therapy.

\section{Methods}

An open randomised controlled trial was carried out at five centres across Australia; participants were recruited from two adult and three paediatric tertiary diabetes clinics. The study protocol received institutional Ethics Committee approval at each of the study sites; written consent was obtained from all participants with additional parent/ guardian consent for participants aged $<18$ years.

Inclusion criteria for participation were: age 13.040.0 years, type 1 diabetes for $>1$ year, use of insulin pump therapy including proficiency with use of a bolus-dose calculator for $>3$ months, $\mathrm{HbA}_{1 \mathrm{c}} \leq 8.5 \%$, reliably performing self-monitoring of blood glucose (SMBG) at least four times daily, and internet access. Willingness to use the subcutaneous sensor component of the system for at least $70 \%$ of the total 3 month study period was a further protocol requirement. Individuals with co-existent medical problems that would interfere with their ability to use the system (e.g. impaired vision), co-existent illness that otherwise predisposes to hypoglycaemia (e.g. adrenal insufficiency) or a history of severe hypoglycaemia while using insulin pump therapy were excluded. Severe hypoglycaemia was defined as an episode of hypoglycaemia resulting in seizure or coma or requiring third-party assistance or the use of glucagon or intravenous glucose for recovery.

Participants were recruited in age- and sex-matched pairs. Each centre generated an alphabetical list of potential participants who were sequentially invited to participate. When an individual gave consent for participation, the next suitable age- and sex-matched person was approached to complete the pair. Each participant was allocated a chronological study number. Once recruited, a pair of participants was entered, in order of study number, into a computergenerated schedule which randomly assigned each of the pair to one of the two study groups. The randomisation schedule was administered centrally; clinicians involved in participant recruitment had no access to the schedule.

Participants in the intervention group received standardised education in use of the integrated sensor-guided pump system and its associated RT features from the same 
instructor across all sites. The system requires calibration using a capillary blood glucose measurement at 2 and $8 \mathrm{~h}$ post insertion and at least 12 hourly thereafter. Hypo- and hyperglycaemic alarms were set to alert the user at sensor glucose levels $<4.5$ and $>12.0 \mathrm{mmol} / 1$, respectively. Participants were also directed to perform confirmatory capillary blood glucose measurements if RT data suggested the need for therapeutic action (e.g. administration of a correction bolus of insulin). RT data were to be uploaded to a web-based management program (Carelink; Medtronic) every 2 weeks. No specific instructive guidelines for interpreting RT data were provided in this study ('patientled' use). Control group participants continued their usual insulin pump therapy and SMBG regimen. Both groups had access to their routine diabetes care team, but no specific additional contact or advice was initiated by the research team over the study period.

Baseline and end-of-study investigations for all participants comprised 6 days of blinded continuous glucose monitoring using the CGMS Gold (Medtronic) and $\mathrm{HbA}_{1 \mathrm{c}}$ measurement. The difference between groups in the proportion of time spent in the target glycaemic range (defined as 4.0-10.0 mmol/l) was the primary outcome of interest. Secondary outcomes included differences in $\mathrm{HbA}_{1 \mathrm{c}}$, proportion of time spent in the hypoglycaemic $(\leq 3.9 \mathrm{mmol} / \mathrm{l})$ and hyperglycaemic $(\geq 10.1 \mathrm{mmol} / \mathrm{l})$ ranges and glycaemic variability. All $\mathrm{HbA}_{1 \mathrm{c}}$ measurements were performed at a central independent DCCT-accredited laboratory (using a Primus CLC330 Affinity Chromatography HPLC analyser [Primus Diagnostics, Kansas City, MO, USA]). Glycaemic variability was analysed using continuous overlapping net glycaemic action (CONGA), which allows for assessment of intra-day variability at repeated time intervals [15]. Sensor accuracy was assessed using mean absolute relative difference (MARD), calculated as the average of the absolute percentage difference between a CGMS sensor reading and a corresponding time-matched capillary glucose reading.

Use of the real-time sensor component of the system by participants in the intervention group was determined from data uploaded to the web-based care management program. The impact of adherence with protocol-stipulated $\geq 70 \%$ overall RT sensor usage throughout the study period on end-of-study $\mathrm{HbA}_{1 \mathrm{c}}$ was also assessed.

Serious adverse events were reported to the study coordinator at each site throughout the study period. Events monitored and recorded included episodes of severe hypoglycaemia or diabetic ketoacidosis, any hospitalisation and device-related complications or malfunction.

This investigator-initiated study was supported by a research grant and provision of equipment from Medtronic Australasia. The manufacturer requested the hypoglycaemic alarm setting of $4.5 \mathrm{mmol} / 1$ (to allow safe recognition of hypoglycaemia despite potentially increased sensor error margin at lower glucose levels), but had no other involvement in study design, data analysis or preparation of the manuscript.

Statistical analyses Our sample size calculation was based on representative CGM data $(n=92)$ from individuals established on insulin pump therapy at one study centre (Royal Children's Hospital, Melbourne, Australia). These data indicated that the mean (SD) proportion of time spent in the target glycaemic range of $4.0-10.0 \mathrm{mmol} / 1$ was $54 \%$ (33\%); a sample of 28 in each group was therefore estimated to have $80 \%$ power to detect a $25 \%$ difference in mean time spent in target range, with a 0.05 two-sided significance level.

Differences in primary and secondary outcome variables between study groups at the end of the study were assessed using: (1) two-sample Student's $t$ tests (unadjusted estimates); and (2) linear regression analysis with baseline value as covariate (adjusted estimates). Use of the RT sensor component of the system was calculated as a percentage of the total study period for each intervention group participant (number of recorded sensor readings divided by total possible sensor readings for the 90 day study period $[288 \times 90] \times 100)$. The impact of adherence with protocol-stipulated use $\geq 70 \%$ on study outcomes was assessed using two-sample Student's $t$ tests. All analyses were intention to treat and performed using Stata 10 (2007; StataCorp LP, College Station, TX, USA). Results of glycaemic outcomes are expressed as mean $\pm \mathrm{SD}$.

\section{Results}

Demographic data, sensor accuracy and adherence to study protocol Sixty-two participants took part in the study; 31 were randomised to each study arm. The study flow diagram is depicted in Fig. 1. Study groups had similar characteristics at baseline (Table 1). Seven participants withdrew from the study: five from the intervention group and two from the control group. The reasons for withdrawal amongst intervention group participants were: burden of recurrent alarms $(n=2)$; significant skin irritation despite use of various different transmitter adhesives $(n=2)$; and inability to maintain prolonged skin adhesion of the sensor because of perspiration $(n=1)$. Two control group participants withdrew consent to undergo end-of-study investigations. End-of-study $\mathrm{CGM}$ and $\mathrm{HbA}_{1 \mathrm{c}}$ data were therefore available for comparison on 26/31 participants in the intervention group and 29/31 in the control group. All participants who withdrew were adolescents (mean age 15.6 years, mean $\mathrm{HbA}_{1 \mathrm{c}}$ 7.6\%). 
Fig. 1 Flow of participants through the study

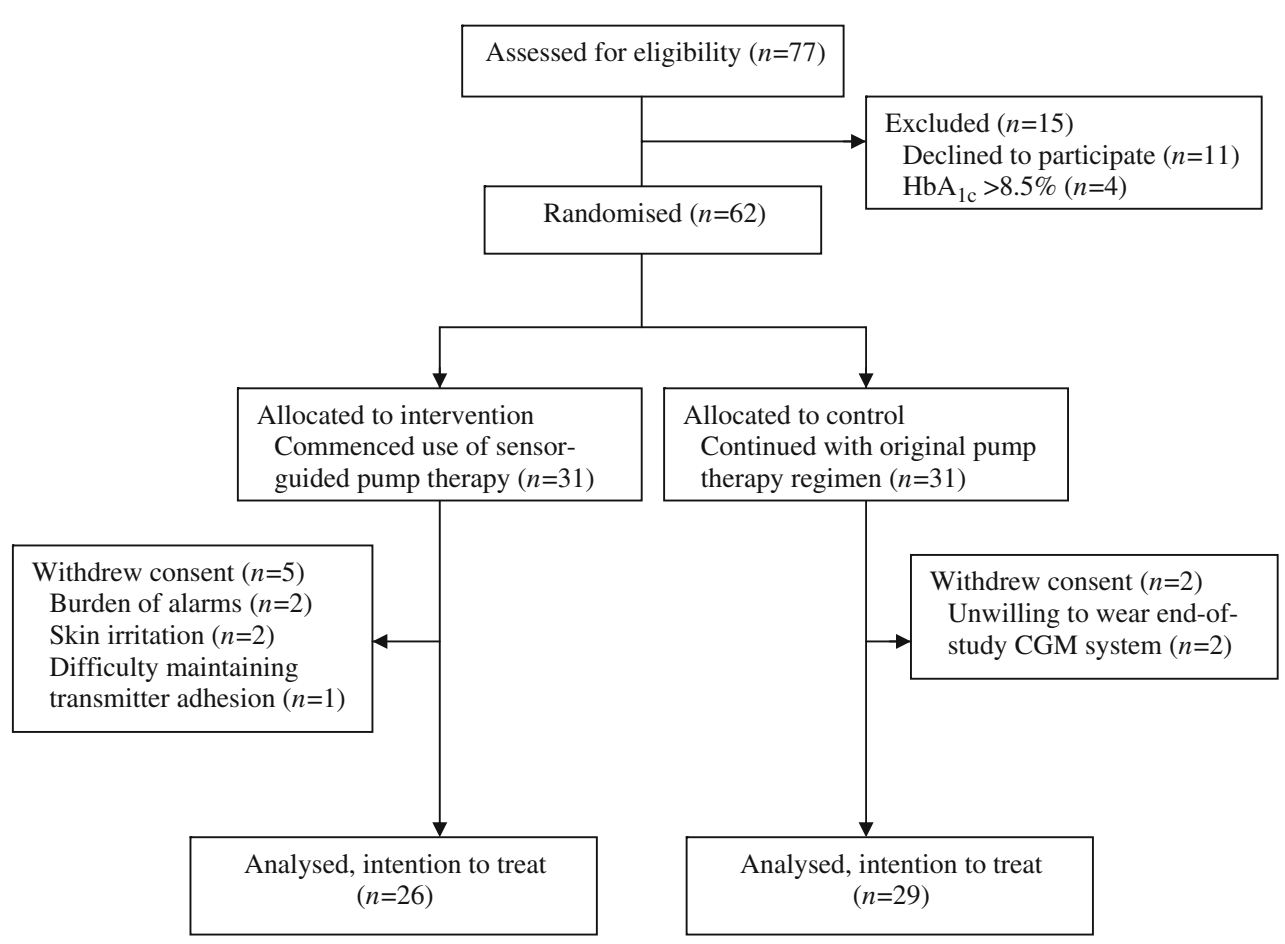

In total, 3,459 paired sensor and blood glucose readings were recorded over the periods of CGM at baseline and the end of the study. Overall mean and median MARD values were $13.3 \%$ and $8.6 \%$, respectively. Variation at different glucose ranges was noted, with mean MARD values of $17.5 \%, 14.5 \%$ and $9.0 \%$ in the predefined hypoglycaemic, target and hyperglycaemic ranges, respectively.

Continuous RT data were uploaded to the web-based management program during the 3 month period by 25/26 participants in the intervention group. One participant did not upload any RT-CGM data but is included in the intention-totreat analyses of principal outcomes as end-of-study investigations were completed. Patterns of RT sensor use varied considerably within the intervention group over the study period. Median (range) time spent using the RT sensor component of the system was $62.5 \%(17.7-93.8 \%)$ of the total 90 day period; $11 / 25$ participants adhered to protocol-stipulated sensor use of $\geq 70 \%$, while $14 / 25$ did not meet this requirement.

Glycaemic outcomes Study outcomes are displayed in Table 2. As shown, there was no difference between study groups in the primary outcome of proportion of time spent in the target glycaemic range over 6 days of CGM at the end of the study. In addition, no between-group difference was found in any of the outcomes of time spent hypo- or hyperglycaemic or glycaemic variation.

Despite the lack of difference in CGM-derived outcome variables, a significant effect on end-of-study $\mathrm{HbA}_{1 \mathrm{c}}$ was

Table 1 Baseline characteristics of all participants randomised to each study group

\begin{tabular}{|c|c|c|c|c|c|c|}
\hline \multirow[t]{2}{*}{ Characteristic } & \multicolumn{3}{|l|}{ Intervention } & \multicolumn{3}{|l|}{ Control } \\
\hline & Total & $\begin{array}{l}13.0- \\
19.0 \text { years }\end{array}$ & $\begin{array}{l}>19- \\
40.0 \text { years }\end{array}$ & Total & $\begin{array}{l}13.0- \\
19.0 \text { years }\end{array}$ & $>19-40.0$ years \\
\hline$n$ (male) & $31(9)$ & $16(6)$ & $15(3)$ & $31(9)$ & $16(6)$ & $15(3)$ \\
\hline Age (years) & $23.4 \pm 8.6$ & $16.2 \pm 1.5$ & $31.1 \pm 5.9$ & $23.0 \pm 8.1$ & $16.2 \pm 1.8$ & $30.4 \pm 5.1$ \\
\hline Duration of diabetes (years) & $11.1 \pm 7.6$ & $7.7 \pm 4.6$ & $14.7 \pm 8.7$ & $9.2 \pm 7.2$ & $5.0 \pm 3.1$ & $13.8 \pm 7.6$ \\
\hline Duration of CSII (years) & $2.4 \pm 2.0$ & $1.6 \pm 1.7$ & $3.2 \pm 1.9$ & $1.9 \pm 1.6$ & $1.7 \pm 1.1$ & $2.2 \pm 2.1$ \\
\hline Total daily dose of insulin $\left(\mathrm{U} \mathrm{kg}^{-1}\right.$ day $\left.^{-1}\right)$ & $0.72 \pm 0.22$ & $0.86 \pm 0.17$ & $0.57 \pm 0.17$ & $0.69 \pm 0.22$ & $0.80 \pm 0.23$ & $0.57 \pm 0.13$ \\
\hline Total daily basal insulin ( $\%$ of total daily dose) & $52.3 \pm 12.9$ & $48.0 \pm 8.7$ & $56.8 \pm 15.3$ & $52.2 \pm 13.3$ & $46.8 \pm 14.5$ & $57.9 \pm 9.5$ \\
\hline $\mathrm{HbA}_{1 \mathrm{c}}(\%)$ & $7.3 \pm 0.6$ & $7.4 \pm 0.7$ & $7.3 \pm 0.5$ & $7.5 \pm 0.7$ & $7.6 \pm 0.6$ & $7.4 \pm 0.8$ \\
\hline
\end{tabular}

Values other than $n$ are expressed as mean \pm SD

CSII, continuous subcutaneous insulin infusion 
Table 2 Glycaemic outcomes

\begin{tabular}{|c|c|c|c|c|c|c|}
\hline \multirow[t]{2}{*}{ Outcome } & \multicolumn{2}{|l|}{ Baseline } & \multicolumn{2}{|l|}{ End of study } & \multirow[t]{2}{*}{ Difference $(95 \% \mathrm{CI})^{\mathrm{a}}$} & \multirow[t]{2}{*}{$p$ value $^{\mathrm{a}}$} \\
\hline & Intervention $^{\mathrm{b}}$ & Control $^{\mathrm{c}}$ & Intervention $^{\mathrm{b}}$ & Control $^{\mathrm{c}}$ & & \\
\hline $\mathrm{HbA}_{1 \mathrm{c}}(\%)$ & $7.3 \pm 0.6$ & $7.5 \pm 0.7$ & $7.1 \pm 0.8$ & $7.8 \pm 0.9$ & $-0.43(-0.19$ to -0.75$)$ & 0.009 \\
\hline \multicolumn{7}{|c|}{ Proportion of time spent (\%): } \\
\hline $4-10 \mathrm{mmol} / 1$ & $62.1 \pm 12.5$ & $58.0 \pm 9.4$ & $57.2 \pm 11.3$ & $53.9 \pm 15.0$ & $1.72(-5.37$ to 8.81$)$ & 0.63 \\
\hline$\leq 3.9 \mathrm{mmol} / 1$ & $9.3 \pm 5.9$ & $10.3 \pm 7.6$ & $9.2 \pm 8.7$ & $9.1 \pm 6.9$ & $0.54(-3.48$ to 4.55$)$ & 0.79 \\
\hline$\geq 10.1 \mathrm{mmol} / 1$ & $28.6 \pm 13.5$ & $31.7 \pm 13.0$ & $33.6 \pm 12.7$ & $37.0 \pm 17.3$ & $-2.18(-10.0$ to 5.69$)$ & 0.58 \\
\hline CONGA $1^{\mathrm{d}}$ & $2.5 \pm 0.4$ & $2.6 \pm 0.5$ & $2.6 \pm 0.5$ & $2.6 \pm 0.6$ & $-0.29(-0.34$ to 0.28$)$ & 0.85 \\
\hline CONGA $2^{\mathrm{d}}$ & $3.6 \pm 0.7$ & $3.7 \pm 0.9$ & $3.8 \pm 0.8$ & $3.8 \pm 1.0$ & $-0.26(-0.45$ to 0.50$)$ & 0.91 \\
\hline $\mathrm{CONGA} 4^{\mathrm{d}}$ & $4.7 \pm 1.1$ & $4.8 \pm 1.3$ & $4.7 \pm 1.0$ & $4.9 \pm 1.2$ & $0.12(-0.51$ to 0.74$)$ & 0.71 \\
\hline
\end{tabular}

Values expressed as mean $\pm \mathrm{SD}$

${ }^{a}$ Difference between end-of-study means, adjusted for baseline values

${ }^{b}$ Data pertain to the $26 / 31$ participants in the intervention group who completed the study

${ }^{c}$ Data pertain to the $29 / 31$ participants in the control group who completed the study

${ }^{\mathrm{d}} \mathrm{CONGA}$ at 1,2 and $4 \mathrm{~h}$ intervals

evident between study groups. End-of-study mean \pm SD $\mathrm{HbA}_{1 \mathrm{c}}$ was $7.1 \pm 0.8 \%$ in the intervention group vs $7.8 \pm$ $0.8 \%$ in the control group, compared with baseline levels of $7.3 \pm 0.6 \%$ and $7.5 \pm 0.7 \%$, respectively. When adjusted for baseline values, mean $\mathrm{HbA}_{1 \mathrm{c}}$ was $0.43 \%$ lower in the intervention group compared with that of the control group ( $95 \%$ CI $0.19-0.75 \%, p=0.009$; Table 2). Overall, a reduction in $\mathrm{HbA}_{1 \mathrm{c}}$ was achieved by $16 / 26$ participants (64\%) who used sensor-guided pump management compared with $5 / 29$ participants $(17 \%)$ who used standard pump therapy. $\mathrm{HbA}_{1 \mathrm{c}}$ levels of $<7 \%$ were also achieved more frequently in the intervention group. Similar proportions in the intervention and control groups (eight [31\%] and ten [34\%] participants, respectively) met this goal at baseline; however, 14/26 (56\%) participants in the intervention group had end-of-study $\mathrm{HbA}_{1 \mathrm{c}}$ levels $\leq 7 \%$, compared with 5/29 (17\%) participants in the control group, $p=0.004$.

Associations between RT sensor usage and glycaemic outcomes Subgroup analysis between those intervention group participants who adhered to protocol-stipulated sensor usage $\geq 70 \% \quad(n=11)$ and those who failed to meet this requirement $(n=14)$ was performed. End-of-study $\mathrm{HbA}_{1 \mathrm{c}}$ was $6.7 \%$ in participants with $\geq 70 \%$ use and $7.4 \%$ in those with $<70 \%$ sensor use; adjusted for baseline values, $\mathrm{HbA}_{1 \mathrm{c}}$ was $0.51 \%$ lower in participants who wore the sensor $\geq 70 \%$ of the total study period $(95 \%$ CI $0.04-0.98 \%$, $p=0.04$; Fig. 2a). The individual change in $\mathrm{HbA}_{1 \mathrm{c}}$ for each intervention group participant is shown in Fig. $2 b$. Only two participants who wore the sensor $\geq 70 \%$ of the total study period did not have improved glycaemic control overall; in each instance, $\mathrm{HbA}_{1 \mathrm{c}}$ was $<7 \%$ at both baseline and the end of the study.
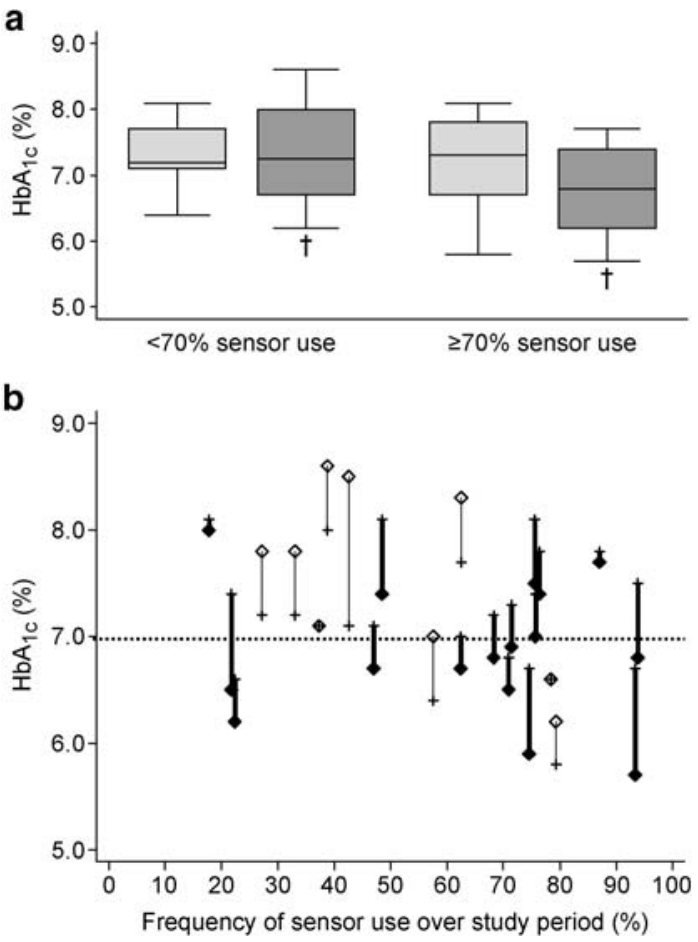

Fig. 2 Effect of frequency of sensor use on $\mathrm{HbA}_{1 \mathrm{c}}$ within the intervention group. a $\mathrm{HbA}_{1 \mathrm{c}}$ grouped according to adherence with protocol-stipulated sensor use $\geq 70 \%$ : baseline results shown as light grey bars; end-of-study results shown as dark grey bars; ${ }^{\dagger} p=0.04$ for the difference between subgroups at the end of the study. $\mathbf{b} \mathrm{HbA}_{1 \mathrm{c}}$ at baseline and 3 months in each individual participant, plotted according to percentage of sensor use. Cross, $\mathrm{HbA}_{1 \mathrm{c}}$ at baseline; black diamond, final $\mathrm{HbA}_{1 \mathrm{c}}$ in participants with improvement in $\mathrm{HbA}_{1 \mathrm{c}}$ over the study period; white diamond, final $\mathrm{HbA}_{1 \mathrm{c}}$ in participants with deterioration in $\mathrm{HbA}_{1 \mathrm{c}}$ over the study period 
Participants with $\geq 70 \%$ sensor use performed a greater number of average SMBG checks per day compared with the less adherent subgroup (5.0/day vs 3.5/day, respectively, $p=0.04)$. The average number of boluses per day was similar between these subgroups (7.0/day vs $7.8 /$ day, respectively, $p=0.5$ ).

Predictors of more frequent use of RT sensor The baseline characteristics of participants in the intervention group were analysed to identify potential predictors of increased sensor use (adherence with $\geq 70 \%$ use). No significant effect of age, duration of diabetes or duration of insulin pump therapy was evident: mean age 22.8 years in those with $\geq 70 \%$ sensor use vs 27.2 years in those with $<70 \%$ use, $p=$ 0.2 ; mean duration of diabetes was 12.0 years and 12.2 years, respectively, $p=0.96$; mean duration of insulin pump therapy use was 1.7 years vs 3.1 years in those with $\geq$ $70 \%$ and $<70 \%$ sensor use, respectively, $p=0.09$.

Adverse events No episodes of severe hypoglycaemia or diabetic ketoacidosis occurred. One participant in the intervention group was admitted to hospital for treatment of new-onset depression; the study protocol was completed nonetheless. Mechanical problems arose: one participant in the intervention group experienced failure of her study insulin pump device necessitating use of her pre-study model until a replacement pump was delivered the following day. It was also necessary to replace radiofrequency transmitters for four participants in the intervention group for whom these devices developed faults that precluded data storage (the problem was identified as repeated 'bad sensor' signals from insertion).

\section{Discussion}

This randomised controlled trial (RCT) demonstrates that self-directed use of sensor-guided pump management is associated with modest improvements in glycaemic control when compared with standard pump therapy in individuals with type 1 diabetes. Participants randomised to use sensorguided management achieved a mean $\mathrm{HbA}_{1 \mathrm{c}}$ level that was $0.43 \%$ lower than that of those who continued standard insulin pump therapy. Sensor usage $\geq 70 \%$ positively influenced glycaemic outcomes.

Previous RCTs of this technology have yielded varying results $[8,10,14,16]$. Two studies have shown improved glycaemic control with use of RT-CGM in patients using either multiple daily injections or insulin pump therapy $[8,10]$. Benefits were limited to individuals $\geq 25$ years old in the most recent study [10]. In contrast, RCTs comparing sensor-guided pump management with standard pump therapy alone have found no between-group difference in
$\mathrm{HbA}_{1 \mathrm{c}}[14,16]$. It is possible that glycaemic benefits seen in participants with suboptimal glycaemic control despite previous intensive management may reflect improved use of pump-related technology (such as bolus-dose calculators) rather than benefits related principally to use of RT-CGM. In addition, most of these studies involved regular clinician review of RT-CGM data and/or clinicianinitiated changes to insulin therapy $[8,10,14]$. It has been noted that regular clinician-patient contact is highly labour intensive [17], and there are no agreed guidelines for incorporating RT-CGM data into routine clinical care.

The specific aim of our study, therefore, was to assess whether individuals with relatively well-controlled diabetes on insulin pump therapy could employ RT-CGM to further improve their own glycaemic control. Sensor-guided management was intentionally 'patient-led'. Participants in both study groups were free to adjust insulin delivery as they deemed necessary; neither group received specific direction on how this should be achieved. The end-of-study difference in $\mathrm{HbA}_{1 \mathrm{c}}$ was due to a combination of improvement in the intervention group and deterioration in the control group. Nonetheless, in light of the absence of a glycaemic threshold for development of microvascular complications in the DCCT [18], the $0.43 \%$ difference in $\mathrm{HbA}_{1 \mathrm{c}}$ and the significant increase in numbers achieving target levels of $\mathrm{HbA}_{1 \mathrm{c}}<7.0 \%$ with use of RT-CGM are clinically meaningful. The non-significant increase in mean $\mathrm{HbA}_{1 \mathrm{c}}$ in the control group over the study period also suggests the relative lack of a 'Hawthorne-effect' related to this protocol. Despite the significant end-of-study difference in $\mathrm{HbA}_{1 \mathrm{c}}$, we were unable to identify differences between groups in any of the CGM-derived glycaemic outcomes. Correlations between CGM data and $\mathrm{HbA}_{1 \mathrm{c}}$ have been described previously [10, 19], but have not been found universally. Despite an end-of-study difference in $\mathrm{HbA}_{1 \mathrm{c}}$ of $0.6 \%$, Deiss et al. [8] found no significant differences in CGM-derived mean glucose, hypo- and hyperglycaemic excursions or area under the curve (D. Deiss, Endokrinologikum, Berlin, Germany, personal communication). These discrepancies may reflect the fact that a 6 day period of continuous glucose monitoring is not consistently representative of longer periods of glycaemic control.

In light of the apparent age effect of sensor-guided management noted in the recent Juvenile Diabetes Research Foundation study [10], we performed post-hoc analyses of age-banded subgroups (13.0-19.0 years and $\geq 19.0$ 40.0 years) in our cohort. Interestingly, significant glycaemic benefit was seen only in the 13.0-19.0 years subgroup in our study. After adjusting for baseline value, there was a $0.6 \%$ between-group difference in the end-of-study $\mathrm{HbA}_{1 \mathrm{c}}$ in this younger subgroup $(95 \% \mathrm{CI}-1.1$ to $-0.1 ; p=0.025)$; no significant difference was observed in older participants 
( $95 \% \mathrm{CI}-0.7$ to $0.2 ; p=0.2$ ). CGM-derived study outcomes were not affected by age. These data should be interpreted with caution as analyses were unplanned; however, they suggest that younger participants may indeed profit from this technology. Notwithstanding this, all participants who withdrew from our study were adolescents.

Our study may include unintentional bias. Selection bias was minimised by use of strict recruitment and randomisation procedures, including allocation concealment. The nature of the intervention prevented participant blinding and this may have led to ascertainment bias. Individuals with poor diabetes control were excluded from this study. Thus, our findings may only be applicable to motivated patients who have pre-existing good control on insulin pump therapy.

RT-CGM may be viewed as a behaviour-modification tool; its application requires understanding and interaction from the user and its success is reliant on the manner in which it is employed. The positive association between overall sensor use and glycaemic benefit in our study population has been noted previously $[7,8,10]$. In our cohort, participants with the greatest sensor use also had a higher mean daily frequency of SMBG, but no difference in bolus frequency was evident. Detailed analyses of alterations in insulin delivery and participant behaviours were, however, limited by the small numbers involved in our study. The key question of how individuals employ this technology to improve their glycaemic control therefore requires further enquiry. We acknowledge that the benefits seen in our study relate to short-term use of 'patient-led' sensor-guided pump management. Further RCTs of longer duration are required to determine the durability of these effects, as frequency of sensor use and behavioural responses to this technology may alter over time.

Recently, high user satisfaction and patient preference for RT-CGM were reported for a cohort with poor glycaemic control using insulin pump therapy [16]. Our findings demonstrate that, with basic education in the use of this technology, some patients with type 1 diabetes can indeed adapt to RT glycaemic information and successfully incorporate it into their diabetes management. Nevertheless, the relatively high rate of participant withdrawal and failure to comply with protocol-stipulated sensor use amongst a motivated patient population suggests that patient-led use of this technology is not universally acceptable and is too high an additional burden for some. In light of the additional expense associated with continuous use of RT-CGM, identification of those most likely to benefit from this technology will be an important focus of future studies.

In conclusion, this RCT has shown that patient-led use of sensor-guided pump management offers additional benefits for glycaemic control when compared with standard insulin pump therapy regimens. Improvement in
$\mathrm{HbA}_{1 \mathrm{c}}$ in such a well-controlled cohort, despite no additional patient-clinician contact over the study period, is clinically significant and has encouraging implications for future use of this technology. Ongoing technological improvements and the introduction of algorithms to guide responses to RT-CGM data are likely to optimise use of this management tool.

Acknowledgements This investigator-initiated study was supported by Medtronic Australasia. The authors would like to thank the following clinicians who were each involved in participant recruitment and co-ordination of study investigations: M. Burgess (St Vincent's Hospital Melbourne, Melbourne, Australia), N. Ratnam (Princess Margaret Hospital for Children, Perth, Australia) and N. Armstrong (The Children's Hospital at Westmead, Sydney, Australia). We are also grateful to I. Goodall and staff at the Division of Laboratory Medicine, Austin Health, Heidelberg, VIC, Australia for assistance with $\mathrm{HbA}_{1 \mathrm{c}}$ measurements for this study.

Duality of interest M. A. O'Connell has received travel and education support from Novo Nordisk and Medtronic Australasia. D. N. O'Neal, T. W. Jones and E. A. Davis have received research support and/or honoraria from Medtronic Australasia. G. R. Ambler has received research support, travel support or honoraria from the following diabetes-related companies: Novo Nordisk, Lilly, SanofiAventis, Animas and Medtronic Australasia. F. J. Cameron has received research support, travel support and honoraria from Novo Nordisk, Lilly and Medtronic Australasia. S. Donath and P. G. Colman have no conflict of interest to disclose.

\section{References}

1. Chase HP, Kim LM, Owen SL et al (2001) Continuous subcutaneous glucose monitoring in children with type 1 diabetes. Pediatrics 107:222-226

2. Ludvigsson J, Hanas R (2003) Continuous subcutaneous glucose monitoring improved metabolic control in pediatric patients with type 1 diabetes: a controlled crossover study. Pediatrics 111:933-938

3. Salardi S, Zucchini S, Santoni R et al (2002) The glucose area under the profiles obtained with continuous glucose monitoring system relationships with $\mathrm{HbA}(\mathrm{lc})$ in pediatric type 1 diabetic patients. Diabetes Care 25:1840-1844

4. Kaufman FR, Gibson LC, Halvorson M, Carpenter S, Fisher LK, Pitukcheewanont P (2001) A pilot study of the continuous glucose monitoring system: clinical decisions and glycemic control after its use in pediatric type 1 diabetic subjects. Diabetes Care 24:2030-2034

5. Chico A, Vidal-Rios P, Subira M, Novials A (2003) The continuous glucose monitoring system is useful for detecting unrecognized hypoglycemias in patients with type 1 and type 2 diabetes but is not better than frequent capillary glucose measurements for improving metabolic control. Diabetes Care 26:1153-1157

6. Yates K, Hasnat Milton A, Dear K, Ambler G (2006) Continuous glucose monitoring-guided insulin adjustment in children and adolescents on near-physiological insulin regimens: a randomized controlled trial. Diabetes Care 29:1512-1517

7. Bailey TS, Zisser HC, Garg SK (2007) Reduction in hemoglobin A1C with real-time continuous glucose monitoring: results from a 12-week observational study. Diabetes Technol Ther 9:203-210 
8. Deiss D, Bolinder J, Riveline JP et al (2006) Improved glycemic control in poorly controlled patients with type 1 diabetes using realtime continuous glucose monitoring. Diabetes Care 29:2730-2732

9. Halvorson M, Carpenter S, Kaiserman K, Kaufman FR (2007) A pilot trial in pediatrics with the sensor-augmented pump: combining real-time continuous glucose monitoring with the insulin pump. J Pediatr 150:103.e1-105.e1

10. The Juvenile Diabetes Research Foundation Continuous Glucose Monitoring Study G (2008) Continuous glucose monitoring and intensive treatment of type 1 diabetes. New Engl J Med 359:1464-1476

11. Diabetes Research in Children Network (DirecNet) Study Group, Buckingham B, Beck RW et al (2007) Continuous glucose monitoring in children with type 1 diabetes. J Pediatr 151:388-393

12. Garg S, Zisser H, Schwartz S et al (2006) Improvement in glycemic excursions with a transcutaneous, real-time continuous glucose sensor: a randomized controlled trial. Diabetes Care 29:44-50

13. Bode B, Gross K, Rikalo N et al (2004) Alarms based on real-time sensor glucose values alert patients to hypo- and hyperglycemia: the guardian continuous monitoring system. Diabetes Technol Ther 6:105-113
14. Hirsch IB, Abelseth J, Bode BW et al (2008) Sensor-augmented insulin pump therapy: results of the first randomized treat-to-target study. Diabetes Technol Ther 10:377-383

15. McDonnell CM, Donath SM, Vidmar SI, Werther GA, Cameron FJ (2005) A novel approach to continuous glucose analysis utilizing glycemic variation. Diabetes Technol Ther 7:253-263

16. Peyrot M, Rubin RR (2009) Patient-reported outcomes for an integrated real-time continuous glucose monitoring/insulin pump system. Diabetes Technol Ther 11:57-62

17. Messer L, Ruedy K, Xing D et al (2009) Educating families on real time continuous glucose monitoring: the Direcnet navigator pilot study experience. Diabetes Educ 35:124-135

18. Anonymous (1996) The absence of a glycemic threshold for the development of long-term complications: the perspective of the Diabetes Control and Complications Trial. Diabetes 45:12891298

19. Deiss D, Hartmann R, Schmidt J, Kordonouri O (2006) Results of a randomised controlled cross-over trial on the effect of continuous subcutaneous glucose monitoring (CGMS) on glycaemic control in children and adolescents with type 1 diabetes. Exp Clin Endocrinol Diabetes 114:63-67 